\title{
Adaptive Heartbeat Modeling for Beat-to-Beat Heart Rate Measurement in Ballistocardiograms
}

\author{
Joonas Paalasmaa, Hannu Toivonen, and Markku Partinen
}

\begin{abstract}
We present a method for measuring beat-to-beat heart rate from ballistocardiograms acquired with force sensors. First, a model for the heartbeat shape is adaptively inferred from the signal using hierarchical clustering. Then, beat-to-beat intervals are detected by finding positions where the heartbeat shape best fits the signal. The method was validated with overnight recordings from 46 subjects in varying setups (sleep clinic, home, single bed, double bed, two sensor types). The mean beat-to-beat interval error was $13 \mathrm{~ms}$ and on average $54 \%$ of the beat-to-beat intervals were detected. The method is part of a home-use e-health system for unobtrusive sleep measurement.
\end{abstract}

Index Terms-heart rate measurement, ballistocardiography, e-health

\section{INTRODUCTION}

$\mathbf{L}$ ONG-TERM measurement of sleep can be used for improving sleep quality and well-being. Bed-installed force sensors are a good solution for long-term measurement, because they cause no discomfort to sleep and function automatically without any actions required from the user.

This paper presents a novel method for detecting beatto-beat heart rate from signals that are measured with such bed-installed force sensors. The method is part of a sleep measurement e-health system where the vibrations of the body are measured with a flexible bed-installed piezoelectric film sensor and sent for analysis to a web service or the user's smartphone (see Section II). In the system, beat-to-beat heart rate measurement is utilized e.g. in estimating stress reactions, sleep stages, and resting heart rate level.

The method we propose is based on ballistocardiography (BCG). Each time the heart beats, the acceleration of blood generates a mechanical impulse that can be measured with a bed-installed force sensor. While BCG-based cardiology research was popular already in the mid-20th century, other methods such as electrocardiography (ECG) practically superseded BCG as a clinical diagnosis method by the early $1980 \mathrm{~s}$ [1]. Recent improvements in measurement technology [2], [3] and applications such as sleep monitoring have made BCG attractive again.

The relation between BCG and ECG is shown in Fig. 1. Each ECG heartbeat consists of a clear spike (the QRS

Manuscript received November 2, 2013; revised February 5, 2014; accepted March 18, 2014. This work was supported in part by Helsinki Doctoral Programme in Computer Science (Hecse) and Finnish Centre of Excellence for Algorithmic Data Analysis Research (Algodan).

J. Paalasmaa is with Beddit Ltd, Espoo, Finland (email: joonas.paalasmaa@beddit.com).

H. Toivonen and J. Paalasmaa are with Department of Computer Science and HIIT, University of Helsinki, Helsinki, Finland.

M. Partinen is with Helsinki Sleep Clinic, Vitalmed Research Centre, Helsinki, Finland and with Department of Clinical Neurosciences, University of Helsinki, Helsinki, Finland.

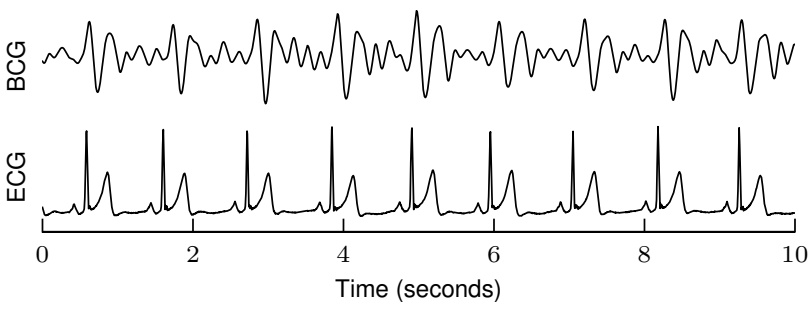

Fig. 1. A 10-second excerpt of synchronized ballistocardiography (top) and electrocardiography (bottom) signals.

complex), which is followed by an impulse in the BCG around 80 ms later [4]. The QRS complex precedes the impulse in the BCG, because electrical activity causes the mechanical contraction of the heart.

The task of detecting beat-to-beat intervals in a BCG signal is much more difficult than detecting those from ECG, because the impulses in BCG are less pronounced and more variable (see below) than the salient shape of the QRS complex. The variability of the BCG heartbeat shape has two aspects. First, the heartbeat shape depends on the physiology of the measured person and the details of the measurement configuration. For example, the shape is different depending on whether the person is lying prone or supine. Second, there is short-term variability in the heartbeat shape that is caused by the mixing of adjacent heartbeats [5] and by respiration [6].

Various methods have been proposed for the measurement of heart rate from BCG signals. Methods that are able to detect individual beat-to-beat intervals, instead of calculating the average heart rate over a time period, are reviewed next.

Various algorithms are based on pre-processing the BCG signal with digital filters such as wavelets, and detecting peaks [7]-[9] or slightly more complex features [10]-[14] (typically a "W" shape) from the signal. The problem with these methods is that they make strong assumptions about the shapes of the heartbeats in the signal. The properties of the BCG signal vary so much in practice that no simple filtering rule can be devised for accurate and reliable beat-to-beat interval detection.

Clustering methods have been proposed for directly detecting heartbeat positions without strong prior assumptions about heartbeat shape [15], [16]. The problem with these methods is that they do not model the region between the detected heartbeat positions. Therefore, it is difficult to infer if two consecutive detected heartbeat positions form a genuine beatto-beat interval or if there is a heartbeat between them that was missed by the clustering procedure. One promising method uses $k$-means clustering to extract a template for the heartbeat shape and detects heartbeats and beat-to-beat intervals with 


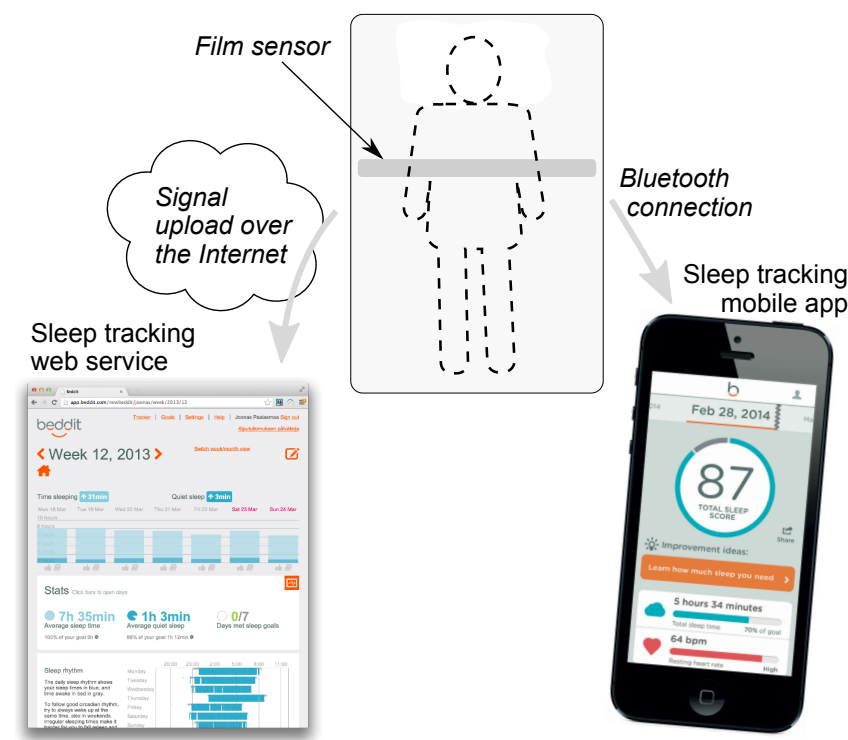

Fig. 2. Overview of the sleep measurement system. Vibrations of the body are measured with a force sensor placed under the body in the bed. The signals are analyzed at a web service or smartphone app and presented to the user.

the template [17].

Another class of methods does not try to detect beat-to-beat intervals by first finding heartbeat positions, but estimates a "fundamental frequency" of short signal segments and infers beat-to-beat intervals that way [18]-[20]. The method by Brüser et al. [20] has been validated with 33 test subjects and can be considered to represent the state of the art.

In the rest of this paper, we present a novel method for detecting beat-to-beat intervals from a BCG signal. The method is based on describing the signal with a simple generative model that takes the physiological variability of the heartbeat shape into account. Using an adaptive model for a single heartbeat, the method is designed to detect heartbeat intervals, i.e., pairs of successive heartbeats. The algorithm is relatively simple and has few heuristics or parameters.

The sleep measurement e-health system where the proposed method is utilized is described in Section II, and the method in Section III. We present a validation with overnight recordings from 46 subjects in Section IV. We conclude with a discussion in Section V.

\section{SLEEP MEASUREMENT SYSTEM}

The beat-to-beat interval detection method is part of a sleep measurement system (see Fig. 2 for an overview). Vibrations of the body are measured with a flexible piezoelectric film sensor, which is installed under the bed sheets. The signal is sent for analysis to a web service over the Internet [21] or to a smartphone over Bluetooth. The signal is analyzed in two stages. First, beat-to-beat intervals, respiratory rate variability [22] and movements are extracted from the force sensor signal. Then, based on this information, the quality of sleep is analyzed. The resulting sleep information is shown to the user with a web interface or smartphone app.

The proposed signal analysis method enables the measurement of nocturnal resting heart rate and heart rate variability.

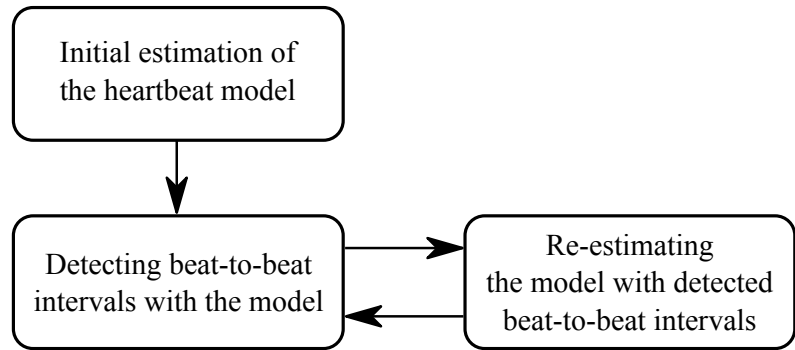

Fig. 3. Overview of the beat-to-beat interval detection process.

In the system, they are utilized e.g. in sleep staging and for measurement of stress reactions during sleep (see e.g. [23][28]). The details are omitted here.

\section{BEAT-TO-BEAT INTERVAL DETECTION METHOD}

\section{A. Purpose and overview}

The purpose of the method is to extract heart rate variability information by finding time intervals between consecutive heartbeat positions (beat-to-beat intervals). For this task, there is no particular reference point in the BCG heartbeat shape that the heartbeat positions need to match. Instead, the method can choose, at run-time, a reference point in the heartbeat shape that it will then use systematically to measure intervals between heartbeats. The temporal location of such a reference point, when identified in the BCG signal, is called a heartbeat position.

A beat-to-beat interval starts at a heartbeat position and ends at the next heartbeat position. This implies that the beat-tobeat interval detection algorithm has to accomplish two tasks: find heartbeat positions for the start and end of the interval, and assure that the start and end positions are consecutive heartbeats (i.e., that there are no missed heartbeats between them).

All this is done in an adaptive manner without existing information about heartbeat positions or heartbeat shapes. The algorithm bootstraps itself as follows (Fig. 3). First, an initial heartbeat shape model is constructed without information about heartbeat positions using clustering. Then, the model is used for detecting beat-to-beat intervals, and periodically updated based on the detected beat-to-beat intervals.

Our implementation is configured to detect beat-to-beat intervals in the range $0.4 \ldots 2.0$ seconds, corresponding to heart rates from 30 to 150 beats per minute. This range is sufficient for most sleep monitoring applications.

Before a measured signal is passed to the beat-to-beat interval detection method, low-frequency activity is removed, to suppress respiration activity. The removed low-frequency activity is extracted by filtering the signal with a Gaussian low-pass filter with 0.1-second standard deviation.

\section{B. Generative model for a BCG signal}

We model the BCG signal as a summation (essentially concatenation) of heartbeat shape vectors [5], [6], [15]. In other words, the signal consists of superimposed shapes of the individual heartbeats (Fig. 4). Due to the oscillating nature of 


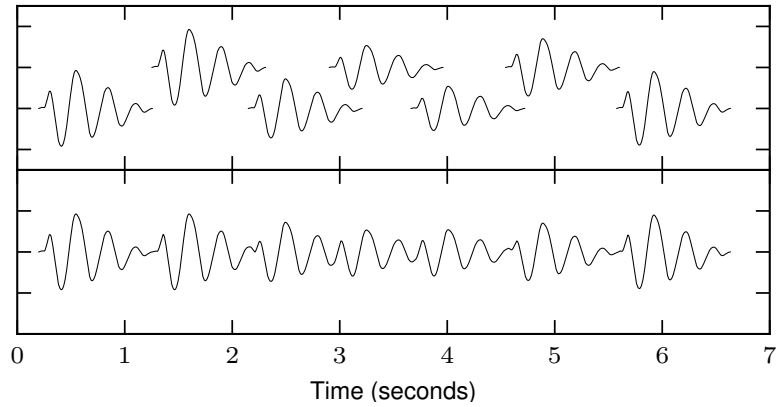

Fig. 4. A synthetic signal example. The individual heartbeat shape vectors $\mathbf{x}_{i}$ are shown in the upper plot (every other heartbeat is offset vertically for clarity). The overall signal, formed by summing the individual heartbeats, is shown in the bottom plot.

the BCG signal, consecutive heartbeat impulses are partially overlapping [5]. The amount of overlap depends on the details of measurement, and an oscillating platform such as a bed makes the overlap longer.

A model for the measured BCG signal that describes the overlap of heartbeats is defined next. Formally, at time $j$, the modelled signal is expressed as

$$
\hat{s}_{j}=\sum_{i=1}^{N} \mathbf{x}_{i}\left[j-t_{i}\right]
$$

where $\mathbf{x}_{i}$ is the shape of the $i$ th heartbeat (a vector), $t_{i}$ is the temporal position of that heartbeat and $\mathbf{x}_{i}[k]$ denotes the $k$ th item of vector $\mathbf{x}_{i}$. We define $\mathbf{x}_{i}[k]=0$ if $k$ is smaller than 0 or larger than the size of vector $\mathbf{x}_{i}$. Note that the individual shape vectors $\mathbf{x}_{i}$ vary between heartbeats (see (2) below).

\section{Respiratory variation of heartbeat shapes}

It has been known since the first ballistocardiography studies [29], [30, p. 191] that the amplitude of the heartbeat shape varies by respiration. We model each individual heartbeat shape vector $\mathbf{x}_{i}$ as

$$
\mathbf{x}_{i}=z_{i} \boldsymbol{\mu}
$$

where vector $\boldsymbol{\mu}$ is a heartbeat shape template (see below) and $z_{i}$ is the amplitude (a scalar) of the $i$ th heartbeat [6].

In our implementation of the algorithm, the length of the heartbeat shape vector $\boldsymbol{\mu}$ is fixed to 2 seconds. This is sufficiently long for accommodating the shape of a single heartbeat fluctuation, at least for the purposes of beat-to-beat interval detection.

We have previously also given a model where heartbeat shape variation is not limited to amplitude, but the shape varies in the direction of a vector [6]. That model is not used here, because the more complex model is difficult to combine with the other parts of this beat-to-beat interval detection method.

\section{Initial estimation of the heartbeat model}

Detection of beat-to-beat intervals consists of two parallel and mutually independent processes: a) estimating the heartbeat shape template $\boldsymbol{\mu}$ based on previously detected beat-to-beat intervals and b) detecting beat-to-beat intervals with $\boldsymbol{\mu}$ (Fig. 3). Their circular dependency is resolved by first detecting a few heartbeat positions using clustering and estimating $\boldsymbol{\mu}$ based on these positions.

A clustering method previously proposed by us [15] is used for finding the initial heartbeat positions. The clustering method is applied on a segment of the signal: within the segment, we look for clusters of short windows (shorter than the beat-to-beat interval) of the signal. A dense cluster of similar windows is most likely to arise when the windows are identically positioned with respect to different heartbeats within the segment.

Windowing and clustering are performed as follows. First, the segment is divided into overlapping 0.4-second windows (minimum allowed beat-to-beat interval) so that each window is centered at an extremum of the derivative of the signal. Fig. 5 illustrates this process with an exemplary 8-second signal segment. The window center positions are shown as upward lines in Fig. 5a and all the 0.4-second signal windows are overlaid in Fig. 5b.

In our implementation, the clustering method is applied to a 15 -second signal segment at a time, and the densest four-item cluster is selected to initially represent the heartbeat shape. This choice is based on experimentation and the idea that about four heartbeats is enough for a rough estimation of the heartbeat shape and 15 seconds typically contains at least four heartbeats not disturbed by movement.

The signal windows are clustered using complete-link agglomerative clustering [15]. Fig. 5c shows the densest (smallest complete-link distance) four-item cluster of signal windows (window positions as downward lines in Fig. 5a). In the estimation of the heartbeat shape template $\boldsymbol{\mu}, 2.5$-second signal windows (shape windows) centered at the four cluster signal windows are first extracted (Fig. 5d). To estimate the length of the heartbeat shape, a heart valve signal (HVS) is calculated (Fig. 5e, computed as the average of squared and low-pass filtered shape windows) [17]. The local minima of the HVS around the center of the segment (vertical lines in Figures 5e-f) determine the length of the heartbeat shape $\mu$ (Fig. 5f).

\section{E. Detecting heartbeat position candidates}

After the heartbeat shape vector $\boldsymbol{\mu}$ has been estimated, beatto-beat intervals can be detected. The detection of a beatto-beat interval consists of finding two consecutive heartbeat positions. The detection of these heartbeat positions is limited to a set of candidate positions (not to be confused with the window positions in the initial estimation of $\boldsymbol{\mu}$ above). These positions are found by computing a sliding cross-correlation between the observed signal $s_{i}$ and the middle 0.4-second segment of the heartbeat shape mean vector $\boldsymbol{\mu}$ [17]. Each local maximum of the cross-correlation is selected as a candidate position, because they match the heartbeat model best. 
This is the author's version of an article that has been published in IEEE Journal of Biomedical and Health Informatics. Changes were made to this version by the publisher prior to publication. The final version of record is available at http://dx.doi.org/10.1109/JBHI.2014.2314144 Copyright (c) 2014 IEEE.

Personal use is permitted. For any other purposes, permission must be obtained from the IEEE by emailing pubs-permissions@iee.org.

(a)

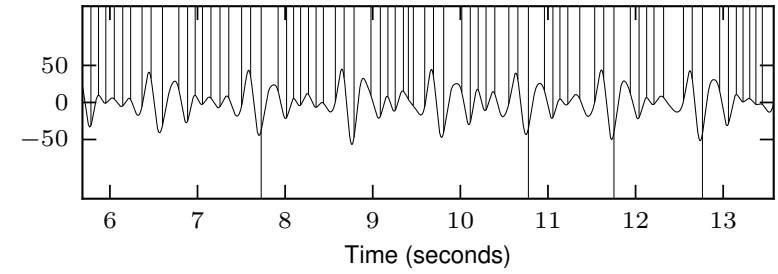

(b)

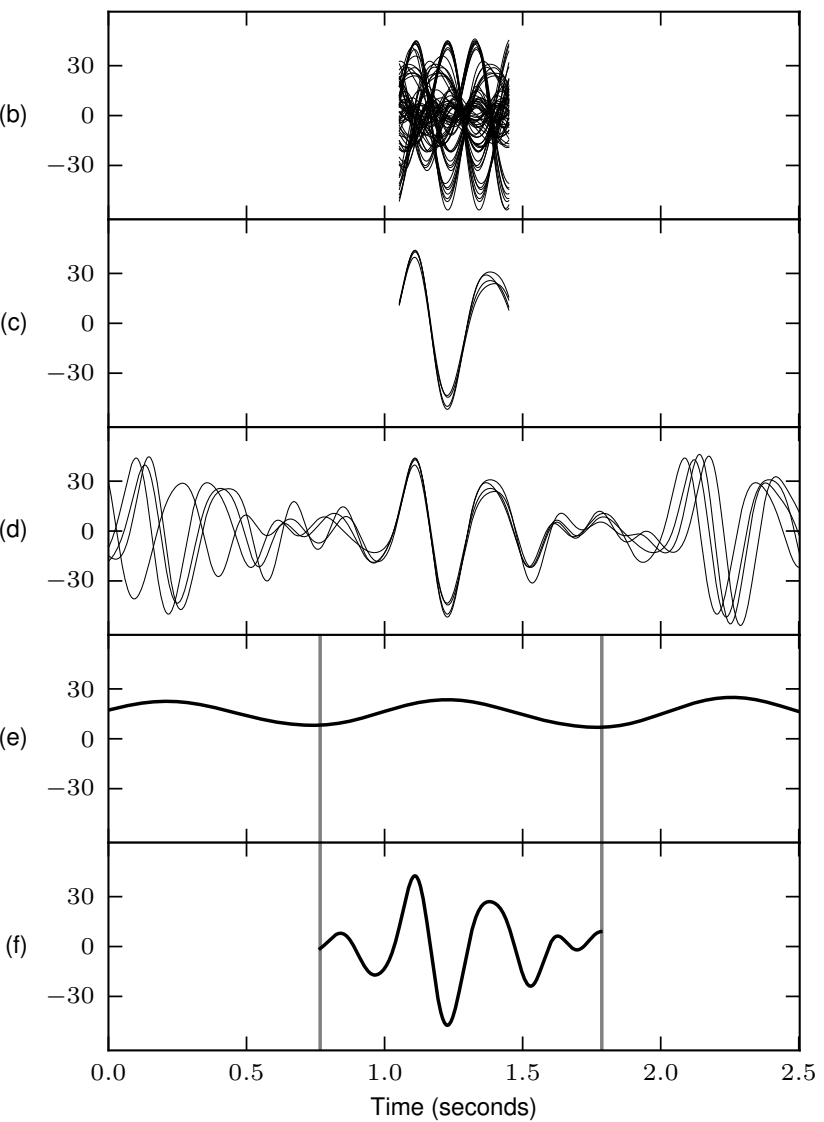

(g)

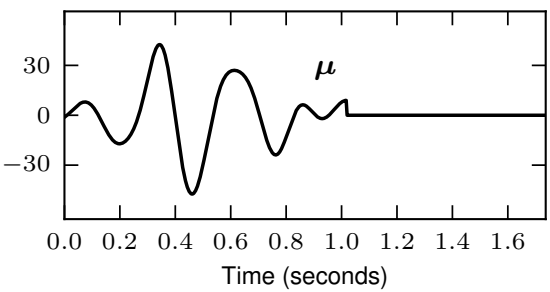

Fig. 5. (a): A 8-second signal segment (shown for improved clarity, although the method uses 15 -second segments). The center positions of the signal windows are shown as vertical upward lines, and of the densest cluster as downward lines. (b): All 0.4-second signal windows overlaid. (c): The signal windows of the densest cluster of four windows. (d): 2.5 -second shape windows that are used for extracting the heartbeat shape model. (e): The computed heart valve signal (HVS). (f): The heartbeat shape is extracted by taking the point-wise mean of the shape windows between the local minima of the HVS. (g): The vector $\boldsymbol{\mu}$ is formed by taking the computed heartbeat shape and filling the rest of the vector with zeros.

\section{F. Detecting beat-to-beat intervals}

Beat-to-beat intervals are detected by finding pairs of consecutive heartbeat positions (denoted by $t_{\mathrm{a}}$ and $t_{\mathrm{b}}$ ) that match the signal well. A potential interval-start heartbeat position $t_{\mathrm{a}}$ is fixed to a candidate position and different candidate positions are tried for the interval-end heartbeat position $t_{\mathrm{b}}$. The algorithm proceeds as follows:

1) Initialize $t_{\mathrm{a}}$ to the first candidate position.

2) Find candidates for $t_{\mathrm{b}}$ in the interval $\left[t_{\mathrm{a}}+0.4 \mathrm{sec}, t_{\mathrm{a}}+\right.$ $2.0 \mathrm{sec}$. The upper and lower limits define the minimum and maximum beat-to-beat interval lengths, respectively.

3) If a $t_{\mathrm{b}}$ candidate results in a small-enough residual (see (4) below) and the amplitudes of the two heartbeats are similar enough (see below), output beat-to-beat interval $\left(t_{\mathrm{a}}, t_{\mathrm{b}}\right)$ and set $t_{\mathrm{a}} \leftarrow t_{\mathrm{b}}$. Otherwise set $t_{\mathrm{a}}$ to be the heartbeat position candidate in the interval $\left[t_{\mathrm{a}}, t_{\mathrm{a}}+2.0 \mathrm{sec}\right]$ with the highest cross-correlation. That position is a good guess for the beginning of a heartbeat interval, as it corresponds to the best-matching heartbeat position in the next 2 -second interval. Return to step 2.

How well the positions match the signal is calculated by constructing a synthetic signal segment based on the positions and the heartbeat model, and computing the difference between the observed and synthetic signals. This computation is visualized in Fig. 6 with two cases. The residual is much higher in the case where the heartbeat interval does not match the signal well.

First, heartbeat vectors $\mathbf{x}_{\mathrm{a}}$ and $\mathbf{x}_{\mathrm{b}}$ are constructed by fitting the heartbeat vector model (2) to the signal at positions $t_{\mathrm{a}}$ and $t_{\mathrm{b}}$. The fitting is done by minimizing the mean-square error between the model (2) and the observed signal $s_{j}$, by adjusting parameter $z$ in (2). The mean-square error is given for the heartbeat position $t_{\mathrm{a}}$ by

$$
\text { average }\left(\left(s_{j}-z_{\mathrm{a}} \times \boldsymbol{\mu}\left[j-t_{\mathrm{a}}\right]\right)^{2}\right)
$$

where $j$ goes from $t_{\mathrm{a}}$ to the end of the heartbeat shape $\left(t_{\mathrm{a}}+\right.$ $2 \mathrm{sec}$ in our implementation). The value of parameter $z_{\mathrm{a}}$ that minimizes the mean-square error is calculated, which gives the synthetic heartbeat vector $\mathrm{x}_{\mathrm{a}}=z_{\mathrm{a}} \boldsymbol{\mu}$. The heartbeat vector for $t_{\mathrm{b}}\left(\mathrm{x}_{\mathrm{b}}\right)$ is constructed in the same way. The reconstructed two-beat region is then obtained as a superposition of $x_{a}$ and $\mathrm{x}_{\mathrm{b}}$.

Then, the modelling error $\epsilon_{j}$ is calculated based on (1), as

$$
\epsilon_{j}=s_{j}-\left(\mathbf{x}_{\mathrm{a}}\left[j-t_{\mathrm{a}}\right]+\mathbf{x}_{\mathrm{b}}\left[j-t_{\mathrm{b}}\right]\right)
$$

over the region $t_{\mathrm{a}} \leq j \leq t_{\mathrm{a}}+\left(t_{\mathrm{b}}-t_{\mathrm{a}}\right) \times 2$ (the two-beat region).

The heartbeat interval $\left(t_{\mathrm{a}}, t_{\mathrm{b}}\right)$ is accepted if the average of the square residual $\epsilon_{j}^{2}$ over the region is below a fixed threshold and the amplitude comparison criterion given below is satisfied. We used $0.2 \times$ average $\left(s_{j}^{2}\right)$ over the region as the threshold.

The amplitudes of $\mathbf{x}_{\mathrm{a}}$ and $\mathbf{x}_{\mathrm{b}}$ are compared to each other when evaluating an interval [15]. If the larger amplitude is more than 2 times the other, the interval is discarded. A large amplitude difference between consecutive heartbeats is physiologically unlikely, so such cases would likely be false 

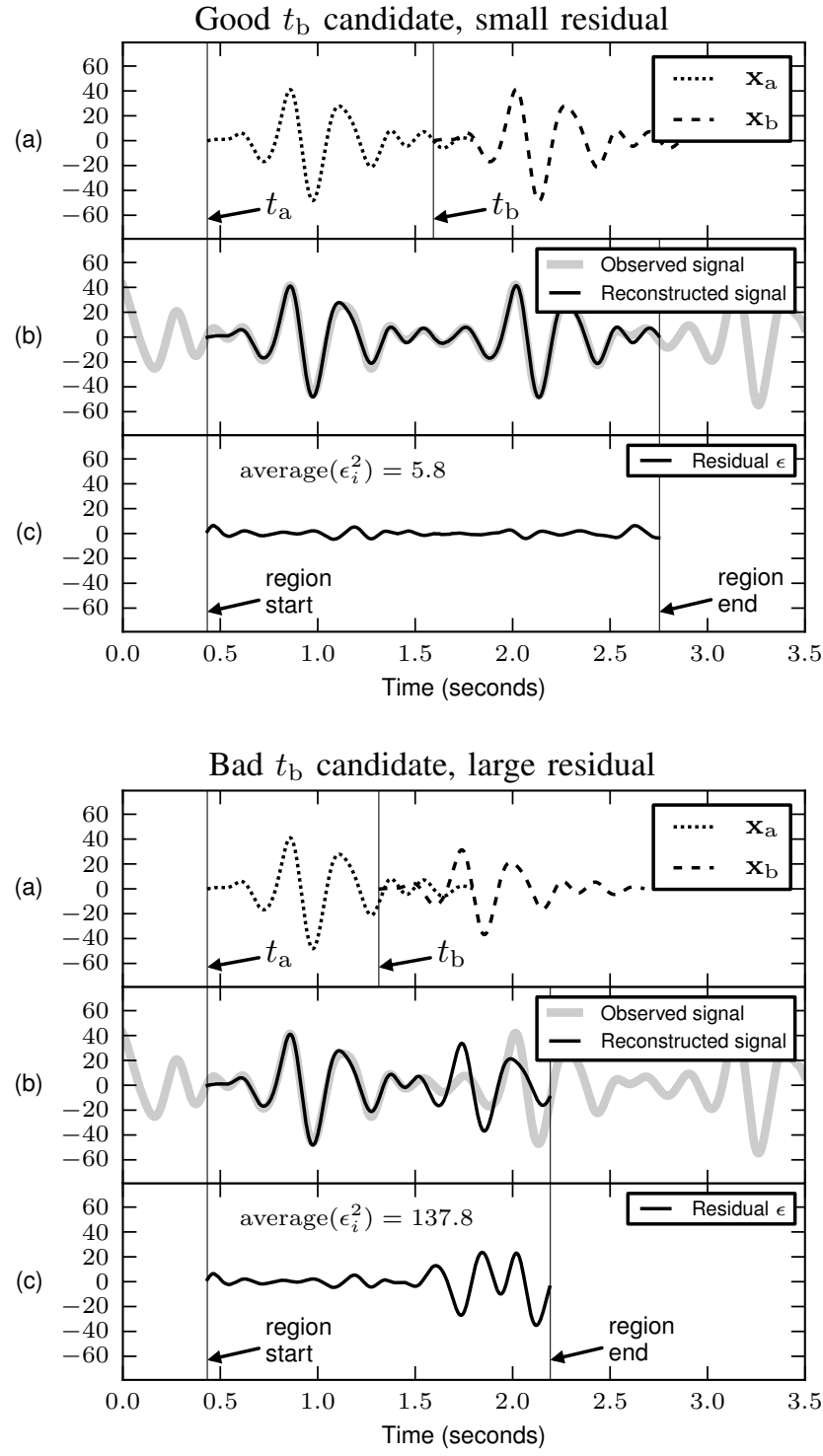

Fig. 6. Beat-to-beat interval evaluation in cases where the residual is low (a $\mathrm{b}, \mathrm{c}$ in the upper panel) and high (a, b, c in the lower panel). (a): Candidate heartbeat vectors $\mathbf{x}_{\mathrm{a}}$ (dotted line) and $\mathbf{x}_{\mathrm{b}}$ (dashed line). (b): The reconstruction of the beat-to-beat interval (black) and the observed signal (gray). (c): The computed residual (4).

detections. A higher threshold might be appropriate with some other sensors, where large amplitude variation occurs naturally.

\section{G. Adjusting the heartbeat model}

The vector $\boldsymbol{\mu}$ is re-estimated every 20 new beat-to-beat interval detections, using the latest 100 detected beat-to-beat interval start positions. Estimation is done with the the method by Inan et al. [5], where ensemble averaging is performed so that the interference of adjacent heartbeats is reduced. The model is updated continuously like this, because the shape of the heartbeat can drift slowly, for example due to changes in average heart rate (for rapid changes in the heartbeat shape, see below). In addition, using more heartbeat positions (up to 100) makes it possible to cancel the interference of adjacent heartbeats on the heartbeat shape model.

\section{H. Accounting for abrupt changes of the heartbeat shape}

Updating $\boldsymbol{\mu}$ as described above handles cases where the heartbeat shape changes slowly. However, changes e.g. in sleeping posture can alter the heartbeat shape so much that it needs to be fully re-initialized. To account for such changes, four independent "instances" of the beat-to-beat interval detection method described in Sections III-A - III-G are run in parallel.

Every 20 seconds, the instance with fewest heartbeat detections in the preceding 20 -second period is re-initialized with the clustering method. From every 20 -second period, beat-tobeat intervals are taken from the instance with most detections in that period.

\section{Post-processing}

The beat-to-beat intervals produced by the above method are post-processed by removing probably incorrect beat-to-beat intervals from data. The median $m$ of the previous 15 detected beat-to-beat intervals is calculated for each new interval. If the new beat-to-beat interval is in range $m / 1.6 \ldots m \times 1.6$ it is accepted and rejected otherwise. These limits have been chosen as a suitable trade-off between 1) detecting as many beat-to-beat intervals as possible and 2) detecting as few incorrect beat-to-beat intervals as possible.

\section{RESULTS}

The performance of the method was tested empirically with overnight recordings from 60 people: 40 patients were measured at a local sleep clinic and 20 volunteers at their homes ${ }^{1}$. At the clinic, the BCG signal was measured with a piezoelectric sensor placed under one bedpost of a standard consumer bed [6]. At homes, a flexible piezoelectric film sensor was placed under the bed sheet, as is done with the e-health application of Section II [21]. In total 46 overnight recordings had a successfully acquired ECG reference and those were used for the validation. A relatively high number of failed measurements was caused by an experimental measurement setup, where ECG-BCG synchronization could not always be established.

The beat-to-beat heart rate detection algorithm of Section III was applied in identical form to signals from both sensors, using the same fixed parameter values. This is a deliberate decision in our validation methodology. We are interested in measuring how robust the method is with different settings and users, not in how well it can be optimized for a particular case since that is not feasible in the intended home use anyway. Systematic experimental evaluation of parameter effects is left for future work.

The running time of our Python implementation of the algorithm on a modern laptop PC is about $0.5 \%$ of the temporal signal length.

\footnotetext{
${ }^{1}$ Human subject data collection was approved by the Coordinating Ethics Committee of the District Hospital of Helsinki and Uusimaa, Helsinki, Finland.
} 
This is the author's version of an article that has been published in IEEE Journal of Biomedical and Health Informatics. Changes were made to this version by the publisher prior to publication. The final version of record is available at http://dx.doi.org/10.1109/JBHI.2014.2314144 Copyright (c) 2014 IEEE. Personal use is permitted. For any other purposes, permission must be obtained from the IEEE by emailing pubs-permissions@ieee.org.

TABLE I

PERFORMANCE OF THE METHOD ACROSS THE TEST SUBJECTS

\begin{tabular}{|c|c|c|c|c|c|c|}
\hline $\begin{array}{l}\text { Age, BMI, sex (F/M), } \\
\text { ensor (bedpost A/film B), } \\
\text { bed (single S/double D) }\end{array}$ & $\begin{array}{l}\text { Mean beat- } \\
\text { to-beat } \\
\text { interval (s) }\end{array}$ & $\begin{array}{c}\text { Coverage } \\
(\%)\end{array}$ & $\begin{array}{c}\text { Precision } \\
(\%)\end{array}$ & $\begin{array}{c}E_{\text {mean }} \\
(\mathrm{ms})\end{array}$ & $\begin{array}{l}E_{95} \\
(\mathrm{~ms})\end{array}$ & $\begin{array}{l}E_{\mathrm{HR}} \\
\text { (bpm) }\end{array}$ \\
\hline $39,19.9, F, A, S$ & 1.01 & 83.50 & 99.94 & 4.8 & 12.6 & 0.36 \\
\hline $71,20.8, F, A, S$ & 1.11 & 94.00 & 99.84 & 5.1 & 10.9 & 0.29 \\
\hline $58,21.3, \mathrm{~F}, \mathrm{~A}, \mathrm{~S}$ & 1.10 & 88.69 & 99.91 & 5.9 & 14.9 & 0.25 \\
\hline $64,27.1, \mathrm{M}, \mathrm{A}, \mathrm{S}$ & 1.26 & 83.70 & 99.86 & 7.2 & 19.0 & 0.30 \\
\hline $33,25.9, \mathrm{M}, \mathrm{A}, \mathrm{S}$ & 1.02 & 82.01 & 99.96 & 7.4 & 20.2 & 0.48 \\
\hline $33,25.7, \mathrm{M}, \mathrm{A}, \mathrm{S}$ & 1.13 & 90.49 & 99.89 & 7.4 & 17.5 & 0.39 \\
\hline $43,25.0, \mathrm{~F}, \mathrm{~B}, \mathrm{~S}$ & 1.03 & 63.61 & 99.94 & 7.4 & 18.2 & 0.69 \\
\hline $29,21.5, \mathrm{M}, \mathrm{A}, \mathrm{S}$ & 1.08 & 88.42 & 99.72 & 8.1 & 20.3 & 0.59 \\
\hline $72,30.0, \mathrm{~F}, \mathrm{~A}, \mathrm{~S}$ & 1.06 & 40.63 & 99.31 & 8.1 & 18.6 & 0.33 \\
\hline $35,19.9, \mathrm{M}, \mathrm{A}, \mathrm{S}$ & 1.23 & 70.44 & 99.49 & 8.2 & 19.5 & 0.75 \\
\hline $29,26.7, F, A, S$ & 0.72 & 54.42 & 99.41 & 8.2 & 18.8 & 0.81 \\
\hline $65,40.6, \mathrm{M}, \mathrm{B}, \mathrm{D}$ & 0.97 & 20.76 & 99.74 & 8.4 & 18.9 & 1.83 \\
\hline $50,24.1, \mathrm{M}, \mathrm{A}, \mathrm{S}$ & 1.05 & 69.01 & 99.70 & 8.6 & 21.5 & 0.99 \\
\hline $32,20.4, \mathrm{M}, \mathrm{B}, \mathrm{D}$ & 0.90 & 43.27 & 99.54 & 8.7 & 19.3 & 0.61 \\
\hline $51,23.8, \mathrm{M}, \mathrm{A}, \mathrm{S}$ & 1.15 & 77.94 & 99.74 & 8.8 & 20.4 & 0.61 \\
\hline $28,20.2, \mathrm{M}, \mathrm{B}, \mathrm{D}$ & 1.00 & 31.60 & 99.92 & 9.0 & 21.2 & 0.75 \\
\hline $39,19.6, F, A, S$ & 1.04 & 91.10 & 99.74 & 9.1 & 22.6 & 0.24 \\
\hline $67,28.9, F, A, S$ & 1.05 & 50.87 & 99.28 & 9.6 & 25.6 & 0.89 \\
\hline $31,22.8, \mathrm{M}, \mathrm{A}, \mathrm{S}$ & 1.31 & 63.48 & 99.77 & 10.2 & 25.6 & 0.34 \\
\hline $30,34.7, \mathrm{M}, \mathrm{A}, \mathrm{S}$ & 0.99 & 67.28 & 99.84 & 10.5 & 25.2 & 0.94 \\
\hline $56,26.8, \mathrm{~F}, \mathrm{~B}, \mathrm{~S}$ & 0.99 & 15.97 & 99.08 & 1.0 & 27.3 & 0.88 \\
\hline $41,-, \mathrm{F}, \mathrm{B}, \mathrm{D}$ & 1.03 & 47.91 & 99.79 & 11.0 & 24.2 & 0.45 \\
\hline $20,21.3, \mathrm{~F}, \mathrm{~A}, \mathrm{~S}$ & 1.10 & 80.66 & 99.18 & 11.6 & 22.2 & 1.12 \\
\hline $52,24.0$ & 1.0 & 86.00 & 99.89 & 11.6 & 35.9 & 0.34 \\
\hline $57,29.8, \mathrm{~F}, \mathrm{~B}, \mathrm{D}$ & 0.92 & 8.19 & 98.52 & 11.7 & 23.1 & 0.97 \\
\hline $42,19.8, \mathrm{M}, \mathrm{B}, \mathrm{S}$ & 1.12 & 39.47 & 99.98 & 12.6 & 32.7 & 0.74 \\
\hline $27,26.9, \mathrm{M}, \mathrm{B}, \mathrm{S}$ & 1.08 & 53.72 & 99.39 & 12.8 & 26.1 & 1.13 \\
\hline $61,27.2, \mathrm{M}, \mathrm{A}, \mathrm{S}$ & 0.95 & 41.74 & 98.29 & 13.0 & 29.7 & 0.52 \\
\hline $46,20.1, \mathrm{~F}, \mathrm{~A}, \mathrm{~S}$ & 1.07 & 52.36 & 98.87 & 3.1 & 27.4 & 0.55 \\
\hline $45,31.3, \mathrm{M}, \mathrm{B}, \mathrm{D}$ & & & 99.3 & 13.3 & 30.8 & 1.29 \\
\hline $72,26.0, \mathrm{~F}, \mathrm{~A}, \mathrm{~S}$ & 1.03 & 48.46 & 98.84 & 13.8 & 30.9 & 0.72 \\
\hline $55,-, \mathrm{M}, \mathrm{A}, \mathrm{S}$ & 0.91 & 46.65 & 98.79 & 14.0 & 35.8 & 0.92 \\
\hline $58,30.1, \mathrm{~F}, \mathrm{~A}, \mathrm{~S}$ & 1.00 & 72.77 & 98.75 & 14.2 & 30.3 & 0.64 \\
\hline $54,30.8, \mathrm{M}, \mathrm{A}, \mathrm{S}$ & 1.07 & 73.95 & 99.32 & 14.9 & 33.0 & 0.39 \\
\hline $57,31.0, \mathrm{M}, \mathrm{A}, \mathrm{S}$ & & 52.17 & 98.82 & 15.2 & 33.0 & 0.72 \\
\hline $60,27.5, \mathrm{M}, \mathrm{A}, \mathrm{S}$ & 1.00 & 50.23 & 97.89 & 15.4 & 36.5 & 0.68 \\
\hline $55,32.8, \mathrm{M}, \mathrm{A}, \mathrm{S}$ & 0.93 & 41.50 & 97.68 & 15.6 & 31.2 & 0.84 \\
\hline $46,18.4, \mathrm{~F}, \mathrm{~A}, \mathrm{~S}$ & & & 97.92 & 15.8 & 36.9 & 0.79 \\
\hline $38,29.6, \mathrm{M}, \mathrm{A}, \mathrm{S}$ & 1.29 & 77.09 & 99.06 & 16.4 & 34.7 & 0.55 \\
\hline 71, 29.4, M, A, S & 0.97 & 21.89 & 96.60 & 18.6 & 44.2 & 1.31 \\
\hline $38,29.4, \mathrm{M}, \mathrm{A}, \mathrm{S}$ & 0.92 & 17.34 & 96.89 & 23.4 & 54.3 & 2.10 \\
\hline $49,32.3, \mathrm{M}, \mathrm{A}, \mathrm{S}$ & 1.13 & 33.19 & 97.09 & 24.0 & 47.1 & 1.06 \\
\hline $52,40.4, \mathrm{~F}, \mathrm{~A}, \mathrm{~S}$ & 0.97 & 19.03 & 92.67 & 28.1 & 215.8 & 0.96 \\
\hline $74,32.2, \mathrm{M}, \mathrm{A}, \mathrm{S}$ & 1.14 & 23.95 & 94.84 & 30.1 & 117.5 & 2.53 \\
\hline $51,29.3, \mathrm{M}, \mathrm{A}, \mathrm{S}$ & 0.93 & 23.34 & 96.67 & 32.6 & 69.2 & 0.48 \\
\hline $54,30.1, \mathrm{M}, \mathrm{A}, \mathrm{S}$ & 1.03 & 28.22 & 94.91 & 33.9 & 101.2 & 0.79 \\
\hline Total averages & 1.04 & 54.07 & 98.77 & 13.22 & 35.26 & 0.78 \\
\hline
\end{tabular}

To measure the accuracy of the heart rate detection method, for each beat-to-beat interval detected from the BCG signal, a corresponding reference beat-to-beat interval was sought from the reference ECG signal. The resulting differences between BCG and ECG beat-to-beat intervals from the whole study are visualized in Fig. 7. The ECG beat-to-beat detections were

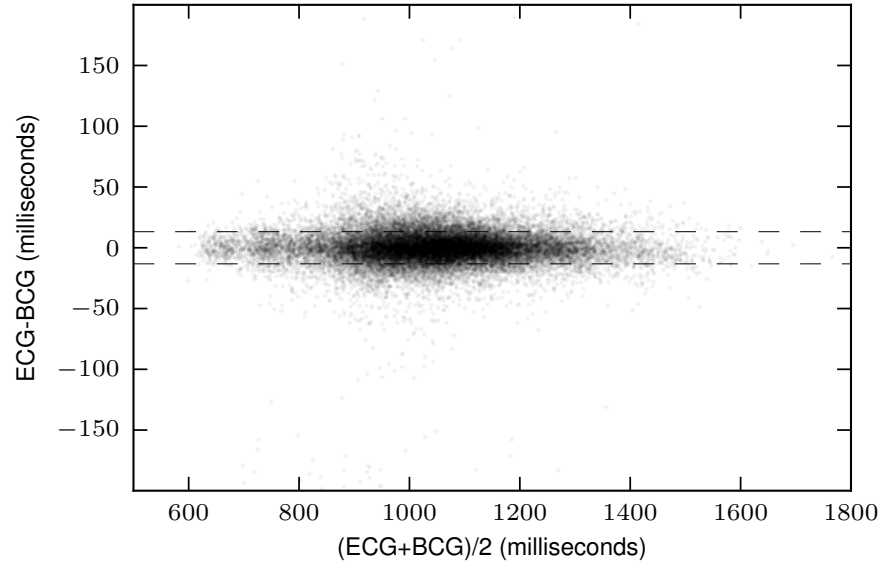

Fig. 7. Bland-Altman plot of the difference between the detected BCG beatto-beat intervals and the ECG reference. Out of the 770676 detected beat-tobeat intervals, 30000 have been randomly selected to make the plot easier to read. The upper dashed horizontal line denotes the average $E_{\text {mean }}$ statistic across all subjects (the other line is its negation).

manually annotated, to make sure that BCG-ECG differences are not caused by ECG artifacts.

The performance of the method is evaluated with the following statistics, computed separately for each test subject (Table I). Coverage is the ratio between the number of detected BCG intervals and the number of detected ECG intervals. Beat-to-beat detections are classified into correct and incorrect detections using a 30 millisecond limit. The ratio precision $=$ correct/(correct + incorrect $)$ is then calculated to give a rough estimate of how large share of the detected beat-to-beat intervals are correct. Based on the absolute differences between ECG and BCG beat-to-beat intervals, the mean ( $\left.E_{\text {mean }}\right)$ and 95th percentile $\left(E_{95}\right)$ of the difference are computed. The mean absolute error of average heart rate measurement $\left(E_{\mathrm{HR}}\right)$ is also calculated, where averaging is performed in 30 -second windows for both BCG and ECG.

The performance of the method varies by test subject. The subjects have different degrees of cardiac problems and sleeping disorders (mainly movement disorders and respiratory problems). This causes the signal quality to vary greatly: the signals are very clear in some cases and full of movement artifacts and other distortion in other cases.

There is large variability e.g. in the coverage parameter. In the best case, $94.0 \%$ of the heartbeats have been detected, whereas in the worst case, only $8.19 \%$ have been detected (average: $54.1 \%$ ). A cause for these coverage differences is visualized in Fig. 8, where typical signal excerpts from highcoverage and low-coverage measurements are shown. The clarity of heartbeat shapes is very different across the excerpts, with discernible heartbeats resulting in better coverage.

We studied how properties of the subject and the measurement setup affect the performance of the method. There is a positive correlation between BMI and $E_{\text {mean }}$ and a negative correlation between BMI and coverage (Table II). Those correlations are statistically significant (likely not caused by chance) according to permutation tests.

The effect of the categorical parameters (sex, single 
This is the author's version of an article that has been published in IEEE Journal of Biomedical and Health Informatics. Changes were made to this version by the publisher prior to publication. The final version of record is available at http://dx.doi.org/10.1109/JBHI.2014.2314144 Copyright (c) 2014 IEEE. Personal use is permitted. For any other purposes, permission must be obtained from the IEEE by emailing pubs-permissions@iee.org.

TABLE II

CORRELATION OF SUBJECT AGE AND BMI WITH METHOD PERFORMANCE

\begin{tabular}{|c|c|c|}
\hline & $\begin{array}{l}\text { Correlation with } \\
E_{\text {mean }} \\
\text { (permutation test } \\
\text { p-value) }\end{array}$ & $\begin{array}{l}\text { Correlation with } \\
\text { coverage } \\
\text { (permutation test } \\
\text { p-value) }\end{array}$ \\
\hline Age & $0.22(0.139)$ & $-0.28(0.057)$ \\
\hline BMI & $0.48(0.001)^{* * *}$ & $-0.51(<0.001)^{* * *}$ \\
\hline
\end{tabular}

${ }^{* * *}$ Significant (permutation testing $p$-value $<0.05$ )

TABLE III

STATISTICAL RELATIONSHIP BETWEEN

SEX, BED TYPE, AND SENSOR TYPE VS. METHOD PERFORMANCE

\begin{tabular}{|c|c|c|c|c|c|}
\hline & Subgroup & $\begin{array}{c}\text { Subgroup } \\
E_{\text {mean }} \\
\text { average }\end{array}$ & $\begin{array}{c}E_{\text {mean }} \\
t \text {-test } \\
\text { p-value }\end{array}$ & $\begin{array}{c}\text { Subgroup } \\
\text { coverage } \\
\text { average }\end{array}$ & $\begin{array}{c}\text { Coverage } \\
t \text {-test } \\
\text { p-value }\end{array}$ \\
\hline \multirow{2}{*}{ Sex } & female & 11.1 & \multirow{2}{*}{0.10} & 58.4 & \multirow{2}{*}{0.34} \\
\hline & male & 14.6 & & 51.3 & \\
\hline \multirow{2}{*}{ Bed } & single & 13.7 & \multirow{2}{*}{0.28} & 57.8 & \multirow{2}{*}{$0.006^{*}$} \\
\hline & double & 10.4 & & 29.2 & \\
\hline \multirow{2}{*}{ Sensor } & bedpost & 14.0 & \multirow{2}{*}{0.18} & 59.4 & \multirow{2}{*}{$0.004^{*}$} \\
\hline & film & 10.6 & & 34.8 & \\
\hline
\end{tabular}

*Significant (t-test p-value $<0.05)$

bed/double bed, bedpost sensor/film sensor) on the performance of the method was tested with two-sample t-test (Table III). The average of $E_{\text {mean }}$ and coverage was computed for each subgroup (e.g. females) and $t$-test was used to determine whether the difference in subgroup averages (e.g. females vs. males) is statistically significant. The only significant effects are: the effect of bed type and sensor type on coverage. The effects of sex, bed, and sensor on $E_{\text {mean }}$ are insignificant.

\section{Discussion AND CONCLUSIONS}

We have presented a new method for detecting beat-tobeat heart rate from ballistocardiograms. It adaptively infers a model for the heartbeat shape from the signal, and then detects beat-to-beat intervals from signal positions matching the heartbeat shape. In our validation study over a range of setups, the method measured beat-to-beat intervals with around 13 ms precision (Table I, column $E_{\text {mean }}$ ). The average error in resting heart rate is less than 1 BPM with most subjects, which enables sufficiently precise average HR measurement for many applications. The e-health system described in Section II utilizes both detailed heart rate variability (HRV) and resting heart rate measurement. If coverage is sufficient, stress reactions of the user are estimated based on HRV, whereas only the resting heart rate number and curve are given if coverage is low.

There is notable inter-subject variability in performance: in the best cases, over $90 \%$ of the heartbeats have been detected with good precision, which should be enough for HRV analyses, whereas in the worst cases, the number of detected heartbeats is sufficient for estimating only the overall trend of resting heart rate. We found out that the measurement setup affects the coverage of the method in a statistically significant way, with film sensor and double bed producing, on average, less coverage (Tables II and III). However, the film sensor and double bed also have better average accuracy (smaller average $\left.E_{\text {mean }}\right)$. As a worst-case example, the cause

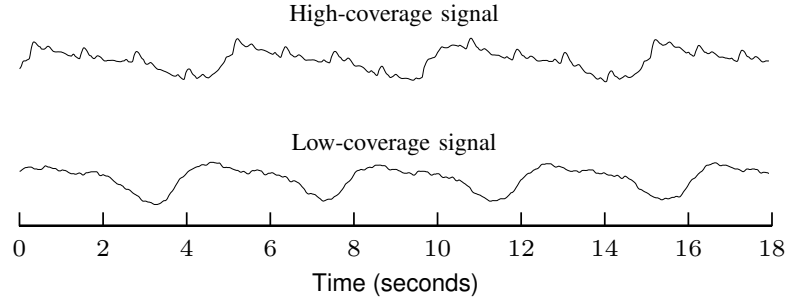

Fig. 8. Excerpts from signals acquired with the film sensor, with differing levels of coverage. The upper signal has $63.61 \%$ coverage and the lower signal $8.19 \%$ coverage. The heartbeat shapes are much cleared in the upper signal. Respiration is the low-frequency phenomenon with around 4-second length.

for the lowest coverage value $8.19 \%$ is not fully known. For some reason, heartbeats shapes in that signal are much less clear than in other cases (Fig. 8). As that measurement has been done at the subject's home, one possible cause is that sensor placement has been suboptimal.

The method contains some parameters, such as modelling residual threshold (Equation (4)), amplitude difference threshold as well as the parameters of the post-processing step. How those parameters should be set depends on the needs of the application and qualities of the signal. Some applications require as many detections as possible, whereas in other cases precision is more important. In this paper we used a set of fixed parameter values, aiming for sufficient accuracy and coverage for sleep monitoring over a range of measurement setups. For other applications, the parameters may need to be optimized differently.

The precision of our method is similar to the beat-to-beat heart rate methods by Brüser et al. [17], [20]. Exact comparison is difficult, because the subject and measurement setup have a large effect on the performance of the method. Our work shows that BCG heart rate measurement is feasible with varying measurement setups and physiological conditions. The presented method is in use in an e-health sleep measurement system (Section II) and it is important that the measurement works with as many people as possible, and in independent home use.

Future work on the subject should study in more detail how the inter-subject variability in the method's performance is related to different measurement setups (bed type, sensor position, sensor installation, ...) and physiological differences between people (BMI, age, diseases, ...). Moreover, the simple post-processing step of removing probably incorrect beat-tobeat intervals should be replaced with a more physiologically justified model. The utility of the method for HRV analysis should be studied by measuring how well BCG and ECG measurements agree on computed HRV parameters.

\section{REFERENCES}

[1] L. Giovangrandi, O. T. Inan, R. M. Wiard, M. Etemadi, and G. T. A. Kovacs, "Ballistocardiography - A method worth revisiting," in Proc. 33rd Annu. Int. Conf. IEEE Eng. Med. Biol. Soc., 2011, pp. 4279-4282.

[2] E. Pinheiro, O. Postolache, and P. Girão, "Theory and developments in an unobtrusive cardiovascular system representation: Ballistocardiography," Open. Biomed. Eng. J., vol. 4, pp. 201-216, 2010.

[3] O. T. Inan, "Recent advances in cardiovascular monitoring using ballistocardiography," in Proc. 34th Annu. Int. Conf. IEEE Eng. Med. Biol. Soc., 2012, pp. 5038-5041. 
This is the author's version of an article that has been published in IEEE Journal of Biomedical and Health Informatics. Changes were made to this version by the publisher prior to publication. The final version of record is available at http://dx.doi.org/10.1109/JBHI.2014.2314144 Copyright (c) 2014 IEEE.

Personal use is permitted. For any other purposes, permission must be obtained from the IEEE by emailing pubs-permissions@iee.org.

[4] M. Etemadi, O. Inan, R. Wiard, G. Kovacs, and L. Giovangrandi, "Noninvasive assessment of cardiac contractility on a weighing scale," in Proc. 31st Annu. Int. Conf. IEEE Eng. Med. Biol. Soc., 2009, pp. 67736776.

[5] O. T. Inan, M. Etemadi, R. M. Wiard, G. T. A. Kovacs, and L. Giovangrandi, "Novel methods for estimating the ballistocardiogram signal using a simultaneously acquired electrocardiogram," in Proc. 31st Annu. Int. Conf. IEEE Eng. Med. Biol. Soc., 2009, pp. 5334-5347.

[6] J. Paalasmaa, "A respiratory latent variable model for mechanically measured heartbeats," Physiol. Meas., vol. 31, no. 10, pp. 1331-1344, 2010.

[7] X. Zhu, W. Chen, T. Nemoto, Y. Kanemitsu, K.-I. Kitamura, K.-I Yamakoshi, and D. Wei, "Real-time monitoring of respiration rhythm and pulse rate during sleep," IEEE Trans. Biomed. Eng., vol. 53, no. 12, pp. 2553-2563, 2006.

[8] M. Brink, C. H. Müller, and C. Schierz, "Contact-free measurement of heart rate, respiration rate, and body movements during sleep," Behav. Res. Methods, vol. 38, no. 3, pp. 511-521, 2006.

[9] R. González-Landaeta, O. Casas, and R. Pallàs-Areny, "Heart rate detection from an electronic weighing scale," Physiol. Meas., vol. 29, no. 8, pp. 979-988, 2008

[10] W. Xu, W. Sandham, A. Fisher, and M. Conway, "Detection of the seismocardiogram W complex based on multiscale edges," in Proc. 18th Annu. Int. Conf. IEEE Eng. Med. Biol. Soc., vol. 3, 1996, pp. 1023-1024.

[11] A. Akhbardeh, B. Kaminska, and K. Tavakolian, "BSeg++: A modified blind segmentation method for ballistocardiogram cycle extraction," in Proc. 29th Annu. Int. Conf. IEEE Eng. Med. Biol. Soc., 2007, pp. 18961899.

[12] P. Smrcka, M. Jirina, K. Hana, and Z. Trefny, "New robust methods for pseudo-period detection in seismocardiographic signal," in Proc. 4th IASTED Int. Conf. Biomed. Eng., 2006, pp. 259-263.

[13] D. Mack, J. Patrie, P. Suratt, R. Felder, and M. Alwan, "Development and preliminary validation of heart rate and breathing rate detection using a passive, ballistocardiography-based sleep monitoring system," IEEE Trans. Inf. Technol. Biomed., vol. 13, no. 1, pp. 111-120, 2009.

[14] D. Friedrich, X. L. Aubert, H. Führ, and A. Brauers, "Heart rate estimation on a beat-to-beat basis via ballistocardiography - A hybrid approach," in Proc. 32nd Annu. Int. Conf. IEEE Eng. Med. Biol. Soc., 2010, pp. 4048-4051.

[15] J. Paalasmaa and M. Ranta, "Detecting heartbeats in the ballistocardiogram with clustering," in Proc. ICML/UAI/COLT 2008 Workshop on Machine Learning for Health-Care Applications, 2008.

[16] L. Rosales, M. Skubic, D. Heise, M. J. Devaney, and M. Schaumburg, "Heartbeat detection from a hydraulic bed sensor using a clustering approach," in Proc. 34th Annu. Int. Conf. IEEE Eng. Med. Biol. Soc., 2012, pp. 2383-2387.

[17] C. Bruser, K. Stadlthanner, S. de Waele, and S. Leonhardt, "Adaptive beat-to-beat heart rate estimation in ballistocardiograms," IEEE Trans. Inf. Technol. Biomed., vol. 15, no. 5, pp. 778-786, 2011.

[18] J. Kortelainen and J. Virkkala, "FFT averaging of multichannel BCG signals from bed mattress sensor to improve estimation of heart beat interval," in Proc. 29th Annu. Int. Conf. IEEE Eng. Med. Biol. Soc., 2007, pp. 6685-6688.

[19] A. Vehkaoja, S. Rajala, P. Kumpulainen, and J. Lekkala, "Correlation approach for the detection of the heartbeat intervals using force sensors placed under the bed posts," J. Med. Eng. Tech., vol. 37, no. 5, pp. 327-333, 2013

[20] C. Brüser, S. Winter, and S. Leonhardt, "Robust inter-beat interva estimation in cardiac vibration signals," Physiol. Meas., vol. 34, no. 2, pp. 123-138, 2013.

[21] J. Paalasmaa, M. Waris, H. Toivonen, L. Leppäkorpi, and M. Partinen, "Unobtrusive online monitoring of sleep at home," in Proc. 34th Annu. Int. Conf. IEEE Eng. Med. Biol. Soc., 2012, pp. 3784-3788.

[22] J. Paalasmaa, L. Leppakorpi, and M. Partinen, "Quantifying respiratory variation with force sensor measurements," in Proc. 33rd Annu. Int. Conf. IEEE Eng. Med. Biol. Soc. IEEE, 2011, pp. 3812-3815.

[23] J. Kortelainen, M. Mendez, A. Bianchi, M. Matteucci, and S. Cerutti, "Sleep staging based on signals acquired through bed sensor," IEEE Trans. Inf. Technol. Biomed., vol. 14, no. 3, pp. 776-785, 2010.

[24] S. Redmond, P. de Chazal, C. O'Brien, S. Ryan, W. McNicholas, and C. Heneghan, "Sleep staging using cardiorespiratory signals," Somnologie, vol. 11, no. 4, pp. 245-256, 2007.

[25] W. Karlen, C. Mattiussi, and D. Floreano, "Improving actigraph sleep/wake classification with cardio-respiratory signals," in Proc. 30th Annu. Int. Conf. IEEE Eng. Med. Biol. Soc., 2008, pp. 5262-5265.
[26] D. Mack, J. Patrie, R. Felder, P. Suratt, and M. Alwan, "Sleep assessment using a passive ballistocardiography-based system: Preliminary validation," in Proc. 31st Annu. Int. Conf. IEEE Eng. Med. Biol. Soc., 2009, pp. 4319-4322.

[27] B. H. Choi, G. S. Chung, J.-S. Lee, D.-U. Jeong, and K. S. Park, "Slowwave sleep estimation on a load-cell-installed bed: A non-constrained method," Physiol. Meas., vol. 30, no. 11, pp. 1163-1170, 2009.

[28] M. Sakakibara, T. Kanematsu, F. Yasuma, and J. Hayano, "Impact of real-world stress on cardiorespiratory resting function during sleep in daily life," Psychophysiology, vol. 45, no. 4, pp. 667-670, 2008.

[29] C. B. Heald and W. S. Tucker, "Recoil curves as shown by the hot-wire microphone," Proc. Roy. Soc. London, Series B, vol. 93, no. 652, pp. 281-298, 1922.

[30] I. Starr and A. Noordergraaf, Ballistocardiography in cardiovascular research. Amsterdam, Netherlands: North-Holland Publishing Company, 1967.

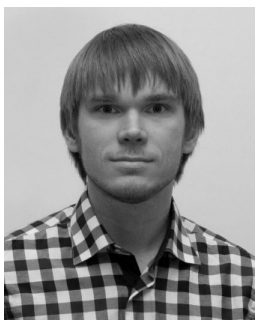

Joonas Paalasmaa received the M.Sc. degree in computer science from Aalto University, Espoo, Finland and the Ph.D. degree in computer science from University of Helsinki, Helsinki, Finland.

$\mathrm{He}$ is Chief Scientist at Beddit Ltd, Espoo, Finland. His research interests include unobtrusive techniques for measuring physiological parameters such as sleep, heart rate and respiration. He has seven granted patents in the fields of mobile devices and biosignal processing

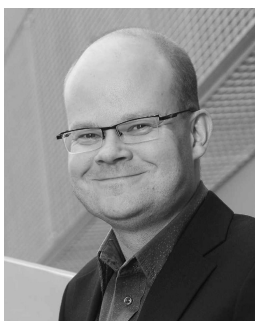

Hannu Toivonen received the M.Sc. and $\mathrm{Ph} . \mathrm{D}$ degrees in computer science from University of Helsinki, Helsinki, Finland.

$\mathrm{He}$ is Professor at Department of Computer Science at University of Helsinki and Vice Director of Algodan, the Finnish Center of Excellence in Algorithmic Data Analysis. His research topics have included data mining and computational methods for data analysis, as well as applications in bioinformatics, genetics, ecology, and mobile communications. The current focus of his research group is computational creativity, i.e., in making computers act creativily or support human creativity, a topic which he combines with data mining.

Professor Toivonen is Program Chair of the 2014 IEEE International Conference on Data Mining.

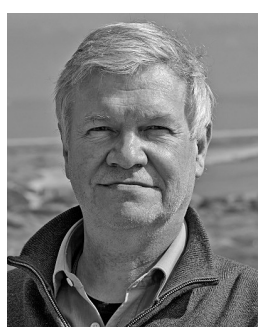

Markku Partinen received the M.D. degree from Université de Montpellier, Montpellier, France and the Ph.D. degree in medicine from University of Helsinki, Helsinki, Finland.

Professor Partinen is Director of Helsinki Sleep Clinic, Vitalmed Research Centre, Helsinki, Finland. $\mathrm{He}$ is Principal Investigator of Sleep Research Program at Department of Clinical Neurosciences, Institute of Clinical Medicine, University of Helsinki. $\mathrm{He}$ is leading the Nordic Consortium studies on narcolepsy and is Chair of the Scientific Committee in the EU Narcolepsy Network as well as Co-chair of the WHO ICF Task Group of Core Sets for Sleep. He has been President of the World Association of Sleep Medicine (WASM) and coordinating secretary of the World Federation of Sleep Research Societies (WFSRS). He is the founder of the Finnish Sleep Research Society (1988) and Finnish Sleep Federation (1998). He has written several books and review articles on sleep medicine. 